\title{
Impressão 3D aplicada ao Ensino de Ciências Básicas da Saúde: onde estamos e onde queremos chegar
}

\author{
Amanda Dalla'Cort Chaves \\ João Miguel Menezes Dutra \\ Cláudio Felipe Kolling da Rocha* \\ Laboratório de Fisiologia e Biofísica \\ - Universidade Feevale
}

\section{Resumo}

As ciências básicas da saúde (CBS) consistem na base da formação do profissional de saúde. A sua compreensão exige abstração e capacidade imaginativa, uma vez que lida com processos subcelulares de difícil visualização. Nesse contexto, novas tecnologias como recursos educacionais impressos em 3D (REs 3D) podem auxiliar na sua compreensão. Este trabalho visa apresentar o atual uso de REs 3D no ensino de CBS e propor atualizações para a sua aplicação no ensino de função. $O$ estudo consiste em uma revisão de literatura seguida de opinião com relato de experiência. $\mathrm{O}$ uso de REs 3D no ensino de CBS é voltado quase que exclusivamente para a morfologia. A adição de tecnologias como microcontroladores e realidade aumentada pode levar os REs $3 \mathrm{~d}$ ao próximo nível, tornando-os adequados ao ensino de função.

Palavras-chave: impressão 3d; ciências básicas da saúde; ensinoaprendizagem

Autor correspondente: claudiodarocha@feevale.br 


\section{D Printing applied to Basic Health Science Education: where we are and what we want to achieve}

\section{Abstract}

The basic health sciences (BHS) are the basis of health professional training. Their understanding requires abstraction and imagination as it deals with difficult-to-view subcellular processes. In this context, new technologies such as educational resources printed in 3D (ERs 3D) can aid in their understanding. The present work aims to present the current use of 3D REs in BHS teaching and propose updates for its application in functionality teaching. The study consists of a review of literature followed by opinion and experience report. The use of 3D ERs in BHS teaching is almost exclusively aimed to morphology. The addition of technologies such as microcontrollers and augmented reality can bring 3D REs to the next level, making them suitable for teaching functionality.

Keywords: 3D printing; basic health sciences; teaching-learning

\section{Impresión 3D aplicada a la Enseñanza de Ciencias Básicas de La Salud: donde estamos y donde queremos llegar}

\section{Resumen}

Las ciencias básicas de la salud (CBS) consisten en la base de la formación del profesional de la salud. Su comprensión requiere abstracción y capacidad imaginativa, ya que se trata de procesos subcelulares de difícil visualización. En este contexto, nuevas tecnologías como recursos educativos impresos en 3D (REs 3D) pueden ayudar en su comprensión. El presente trabajo pretende presentar el actual uso de REs 3D en la enseñanza de CBS y proponer actualizaciones para su aplicación en la enseñanza de función. El estudio consiste en una revisión de literatura seguida de opinión con relato de experiencia. El uso de REs 3D en la enseñanza de CBS se dirige casi exclusivamente a la morfología. La adición de tecnologías como microcontroladores y realidad aumentada puede llevar los REs 3D al siguiente nivel, haciéndolos adecuados a la enseñanza de función.

Palabras clave: impresión 3d; ciencias básicas de la salud; enseñanza y aprendizaje 


\section{Introdução}

As ciências básicas da saúde consistem no alicerce da formação do profissional da saúde. Ciências voltadas para o conhecimento dos processos que regem o funcionamento dos organismos vivos são essenciais para a compressão do processo saúde-doença. Fomentam e sustentam o pensamento crítico e a capacidade de solucionar problemas clínicos, em especial quando estes não seguem o padrão.

No Brasil, as disciplinas que compõem as Ciências Básicas da Saúde (CBS) são definidas pelas diretrizes curriculares por meio de Resoluções específicas publicadas pelo Ministério da Educação. A exemplo disso, de acordo com a Resolução CNE/CES 2, de 18 de fevereiro de 2003, o curso de Biomedicina demanda, além de suas disciplinas específicas, matérias relacionadas a:

[...] II - Ciências Biológicas e da Saúde - incluem-se os conteúdos (teóricos e práticos) de base moleculares e celulares dos processos normais e alterados, da estrutura e função dos tecidos, órgãos, sistemas e aparelhos, bem como processos bioquímicos, microbiológicos, imunológicos e genética molecular em todo desenvolvimento do processo saúde-doença, inerentes à biomedicina.

Da mesma forma, outros cursos da área da saúde também demandam uma base científica circundada pelas disciplinas básicas que envolvem processos fisiológicos em várias escalas. Pensando nisso, essas disciplinas têm grande importância na formação inicial do graduando que, ao longo do seu caminho acadêmico, continuará trabalhando seus conceitos-chave em disciplinas curso-específicas.

O ensino de ciências básicas da saúde enfrenta o desafio de depender da capacidade de abstração dos estudantes, uma vez que, com frequência, aborda processos em escala celular e subcelular (SMITH, 2016). Com a geração atual progressivamente mais dependente de informações visuais e menos treinada para o processo de abstração, o problema de uma formação sólida em ciências básicas da saúde se intensifica (CASTANHA; CASTRO, 2010; FELIX et al., 2009). Ainda nesse contexto, o uso de animais no ensino tem sido fortemente discutido (CANOVA; SILVA; 
GRASSI-KASSISSE, 2015; LIMA; STURN; RIBEIRO, 2018; SARAIVA et al., 2016), impondo a necessidade de renovação dos recursos educacionais utilizados na formação básica dos cursos superiores da área da saúde (CARNIATTTO, 2017).

O presente panorama impele o docente a buscar novas metodologias, as quais podem encontrar suporte em recursos tecnológicos modernos como realidade virtual, realidade aumentada, impressão 3D, aplicativos móveis, simuladores, dispositivos vestíveis e outros. O desenvolvimento de recursos educacionais baseados em tecnologia pode sanar uma deficiência de ensino que nem mesmo o uso de animais foi capaz. Entre os recursos citados, recursos educacionais impressos em 3D vêm ganhando espaço no ensino de ciências básicas da saúde, em especial aquelas com caráter fortemente morfológico. A sua utilização pode auxiliar na visualização didática de estruturas biológicas, da forma mais fidedigna possível, o que antes se limitava somente à imaginação e imagens estáticas ilustrativas (SMITH, 2016).

A tecnologia a que comumente nos referimos como impressão $3 \mathrm{D}$ recebe o nome técnico de manufatura aditiva, sendo sua forma mais popular a modelagem em deposição fundida (Fusion Deposition Modeling FDM) (LEDERLE et al., 2016). No processo FDM, um material termoplástico em forma de filamento sofre derretimento e extrusão, sendo liberado em uma superfície camada por camada, seguindo a geometria do modelo (MANUFACTUR3D, 2018; WONG; HERNANDEZ, 2012). Uma segunda metodologia é a fotopolimerização, da qual o principal exemplo é a esterolitografia. Em tal abordagem, uma resina líquida é solidificada por um laser, criando os pontos que compõem o modelo 3D (MANUFACTUR3D, 2018; WONG; HERNANDEZ, 2012).

Os modelos 3D são construídos a partir de diversos processos envolvendo desenho assistido por computador (CAD) e escaneamento 3D. Os modelos em 3D devem ser submetidos a um software de fatiamento, responsável por separar o desenho em várias camadas de duas dimensões, que serão criadas pela impressora 3D. O formato de arquivo mais comumente utilizado é o STL (Stereolithography) (WONG; HERNANDEZ, 2012). 
Considerando o crescimento do uso de recursos educacionais (REs) impressos em 3D e o potencial de tal tecnologia, o presente trabalho objetiva fomentar a discussão sobre sua aplicação no ensino de ciências básicas da saúde, estando dividido em duas seções. A primeira visa descrever como recursos impressos em $3 \mathrm{D}$ vêm sendo utilizados no ensino de ciências básicas da saúde, a partir de uma revisão da literatura. A segunda visa apresentar nossa visão de como tal aplicação pode ser melhor explorada, utilizando como exemplo os trabalhos do nosso grupo.

\section{Impressão 3D no ensino de ciências básicas da saúde e suas limitações}

Para ilustrarmos o uso atual de recursos impressos em 3D no ensino de ciências básicas da saúde, foi realizada uma pesquisa bibliográfica nas bases de dados PubMed, Scielo e EBSCO. Os termos utilizados na pesquisa foram: "3D printing" and "anatomy" and "education"; "3D printing" and "physiology" and "education"; "3D printing" and "biochemistry" and "education"; "3D printing" and "immunology" and "education"; "3D printing" and "cell biology" and "education" e "3D printing" and "molecular biology" and "education". Os critérios para a seleção dos artigos foram: terem sido publicados nos últimos cinco anos (2013-2018) e abordarem o uso de modelos tridimensionais no ensino das disciplinas já listadas, agrupadas na categoria de CBS. Foram excluídos os artigos que faziam uso de modelos 3D para disciplinas clínico-específicas ou que não tivessem aplicação educacional.

No total, a busca retornou 116 artigos sobre anatomia, 12 sobre fisiologia, 3 sobre bioquímica, 8 sobre biologia molecular, 11 sobre biologia celular e nenhum sobre imunologia. Após a seleção, sobraram 11 artigos sobre anatomia, 1 artigo sobre biologia molecular, 1 artigo sobre biologia celular e 1 artigo correspondente a múltiplas áreas (Bioquímica, Fisiologia, Biologia Molecular e Biologia Celular). A maioria dos estudos retornados para as disciplinas, principalmente para anatomia, foram excluídos por terem foco na área clínica ou em disciplinas clínico-específicas. A principal fonte foi a base de dados PubMed, seguido por Scielo. A base EBSCO não retornou resultados. 
Dos estudos selecionados, foi visto que grande parte dos modelos criados tinham o intuito de ensinar morfologia aos estudantes (AUGUSTO et al., 2016; BACKHOUSE; TAYLOR; ARMITAGE, 2018; BAGLEY; GALPIN, 2015; BANNON et al., 2018; CANTÍN; MUÑOZ; OLATE, 2015; CHEN et al., 2017; DAVENPORT et al., 2017; GARAS et al., 2018; KONG et al., 2016; LI et al., 2018; LIM et al., 2016; MCMENAMIN et al., 2014; SMITH et al., 2018; ) e apenas um dos estudos tinha como objetivo ensinar morfo-funcionalidade (SMITH; JONES, 2018).

Muitas das peças anatômicas foram geradas a partir de dados de tomografia computadorizada, como membro superior e maxilar (MCMENAMIN et al., 2014), segmentos hepáticos (KONG et al., 2016), anatomia cardíaca externa (LIM et al., 2016), estruturas ósseas como o crânio (CHEN et al., 2017), fossa pterigopalatina (BANNON et al., 2018) e demais modelos esqueléticos, incluindo o de animais (LI et al., 2018). Alguns moldes foram criados com o auxílio de um escâner 3D, assim como mostrado por Cantín et al. (2015), que usaram para confeccionar dentes, maxilares e mandíbulas, e Backhouse et al. (2018), que criaram seu modelo por meio do escaneamento de um crânio humano. Bagley e colaboradores (2015) criaram seu artefato baseando-se em uma amostra de tecido muscular, que teve células musculares esqueléticas observadas com o auxílio de microscopia confocal e florescência. O estudo realizado por Augusto et al. (2016) utilizou células sanguíneas derivadas de ratos, observadas por intermédio de microscopia eletrônica, para gerar imagens de leucócitos (neutrófilos e monócitos) que foram utilizadas para confecção e impressão dos modelos 3D. Davenport e cols. (2017) voltaram seu estudo para o campo da biologia molecular, focando em estruturas proteicas construídas a partir das metodologias ball-and-stick (PAUKSTELIS, 2018) e análise da arquitetura TIM barrel (GOLDMAN; BEATTY; LANDWEBER, 2016). Na pesquisa de Smith et al. (2018), um cadáver doado para a Universidade de Sussex foi escaneado para gerar modelos 3D do sistema respiratório e músculo esquelético.

O trabalho morfo-funcional descrito por Smith \& Jones (2018) visou testar modelos 3D de estruturas do pescoço e vias aéreas, disponibilizadas gratuitamente pelo site BodyWorks3D. O estudo utilizou 
a extrusão dupla de filamentos flexíveis e PLA para imprimir cordas vocais e permitir o movimento dos aritenoides na cartilagem cricoide, com o intuito de ilustrar a ação dos músculos intrínsecos da laringe.

Uma importante diferença entre os estudos é a forma de validação que realizaram. Basicamente, os estudos se propõem a validar as estruturas de duas formas distintas: (1) Validação a partir da comparação dos modelos 3D com a estrutura real a qual ele deriva, (2) Validação dos modelos 3D em sala de aula, verificando a opinião e/ou o desempenho dos alunos após seu uso. Do total de 14 estudos selecionados, seis foram validados da primeira forma (AUGUSTO et al., 2016; BANNON et al., 2018; CANTÍN; MUÑOZ; OLATE, 2015; LI et al., 2018; MCMENAMIN et al., 2014; SMITH; JONES, 2018) e 8 da segunda (BACKHOUSE; TAYLOR; ARMITAGE, 2018; BAGLEY; GALPIN, 2015; CHEN et al., 2017; DAVENPORT et al., 2017; GARAS et al., 2018; KONG et al., 2016; LIM et al., 2016; SMITH et al., 2018; STREICHER; KINI; STOFF, 2016).

Dos seis estudos que compararam os modelos $3 \mathrm{D}$ à sua estrutura de origem, quatro concluíram que o método gera estruturas precisas, ou seja, com características muito semelhantes à original (AUGUSTO et al., 2016; BANNON et al., 2018; CANTÍN; MUÑOZ; OLATE, 2015; MCMENAMIN et al., 2014). No entanto, o estudo de Li et al. (2018) verificou que todas as características, exceto o forame nutriente na amostra óssea original, puderam ser identificadas nos modelos digitais e impressos em 3D. O estudo de Smith \& Jones (2018), em contrário à maior parte dos estudos, indica que as limitações do método estão ligadas à qualidade, precisão e estabilidade dos modelos. Logo, a maior parte dos estudos demonstra que os modelos 3D são representações fidedignas da estrutura de interesse da qual derivam.

Dos oito estudos que testaram os modelos 3D em ambiente acadêmico, três relatam aumento do interesse em aprender (BAGLEY; GALPIN, 2015; DAVENPORT et al., 2017; SMITH et al., 2018). Além disso, Lim et al. (2016) verificaram que o uso de modelos 3D avaliados isoladamente conferiu benefícios ao aprendizado anatômico em relação ao uso de materiais cadavéricos ou em combinação com eles. $\mathrm{O}$ estudo realizado por Backhouse et al. (2018) verificou que os participantes 
preferiram o modelo 3D em comparação com os recursos tradicionais de aprendizagem, acreditando que o modelo ajudou no entendimento e visualização, e que aumentou sua confiança para aplicar esse conhecimento. A maior parte dos estudos que aplicaram esse tipo de validação sugere que os modelos 3D sejam utilizados em conjunto com os modelos tradicionais de ensino e de prática (CHEN et al., 2017; GARAS et al., 2018; KONG et al., 2016). Ademais, Kong e colaboradores (2016) validaram os modelos também com professores. Para isso, os pesquisadores desenvolveram três tipos de modelos de segmentos hepáticos a serem avaliados por eles a partir das seguintes variáveis: condição anatômica, qualidade, cor, tato e satisfação geral com o modelo. A validação com os professores serviu como um jeito de avaliar as estruturas com um olhar técnico-científico e escolher o melhor modelo, entre os três criados, para ser utilizado para a aprendizagem sobre o assunto. Apesar de alguns estudos verificarem um melhor desempenho e interesse dos alunos com o uso dos modelos 3D, a maior parte deles sugere seu uso de maneira conjunta aos métodos de ensino tradicionais.

A maior parte dos modelos tem sido utilizada apenas com foco morfológico, ou seja, para demonstrar a forma de estruturas de interesse. Isso pode ser explicado pela densidade de estudos na área anatômica, que estuda a morfologia de órgãos e sistemas como foco principal. Para as disciplinas de CBS, é importante que o aluno saiba reconhecer estruturas (morfologia), mas também é necessário que ele saiba relacioná-las entre si (função), o que tem sido pouco explorado na literatura.

\section{Recursos impressos em 3D para o ensino de processo e função}

Recursos impressos tridimensionalmente têm sido utilizados com frequência para o ensino de aspectos morfológicos, como já descrito. Relatos do seu uso para o ensino de função, o que requer modelos dinâmicos capazes de demonstrar a inter-relação entre processos e sua temporalidade, não são encontrados facilmente. Com isso em mente, nesta segunda etapa nos ateremos a diferentes abordagens que possam ser implementadas com o objetivo de obtermos REs 3D dinâmicos 
que possam ser utilizados para o ensino de função e processo, não se restringindo apenas ao ensino da forma.

\section{Modelagem em software de desenho técnico para produção de peças articuladas}

Entre os diferentes softwares que podem ser utilizados para a criação de modelos em 3D, podemos diferenciar os que permitem uma modelagem mais orgânica e aqueles que seguem a modelagem técnica, de peças múltiplas com dimensões precisas. Na primeira categoria, se enquadram softwares como o Autodesk 3DS Max, Pixologic Z Brush e Pixologic Sculptris. No segundo grupo, possuímos softwares tradicionalmente utilizados para engenharia e projetos de peças, como o Autodesk Fusion 360 e Autocad e SolidWorks da Dessault Systemes. Os softwares para modelagem orgânica são os mais frequentemente utilizados na construção de modelos biológicos que visam ao ensino de morfologia. Embora faça sentido o uso de softwares de modelagem orgânica para criação de modelos biológicos, quando pensamos na elaboração de recursos educacionais que abordem função de forma dinâmica, o uso de softwares como Fusion 360 nos permite a modelagem de peças móveis, articuladas e interativas, favorecendo a visualização da função.

Na Figura 1, temos o exemplo de um modelo articulado desenvolvido pelo nosso grupo, que objetiva o ensino dos processos envolvidos no transporte de moléculas através da membrana das células. Esse tema tem como desafio a necessidade de visualização de processos dinâmicos que ocorrem em escala subcelular. Existem múltiplos mecanismos envolvidos, dependendo do tipo de molécula e da distribuição do soluto no meio. Ao realizarmos o projeto utilizando um software CAD voltado para projeto de peças, pudemos elaborar componentes articulados que representem os pontos essenciais do processo, ilustrando as diferenças entre os diversos mecanismos. 
A)

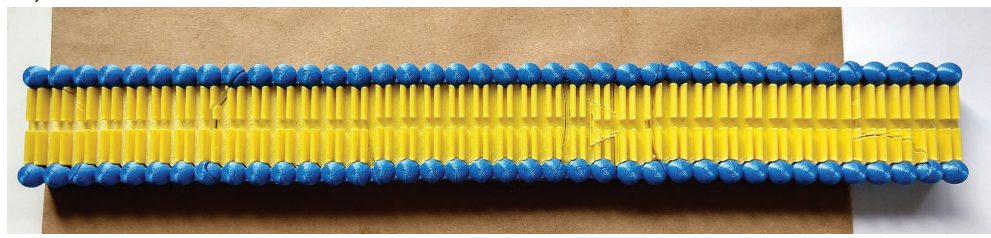

B)

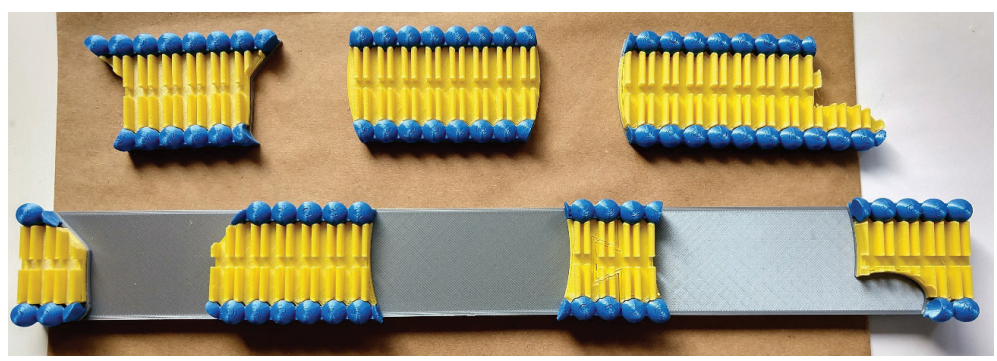

C)

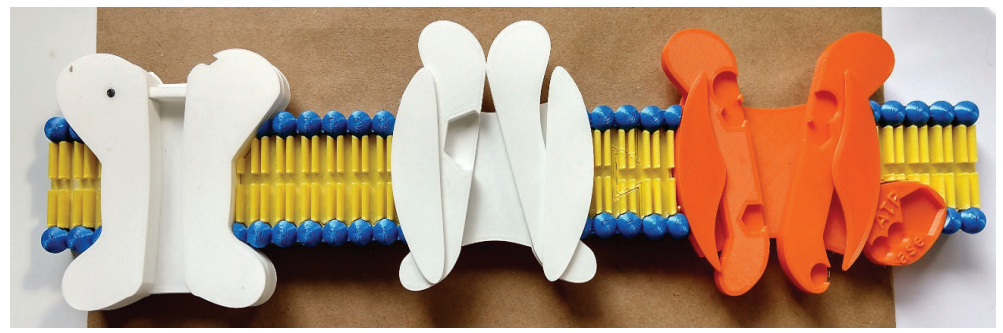

D)

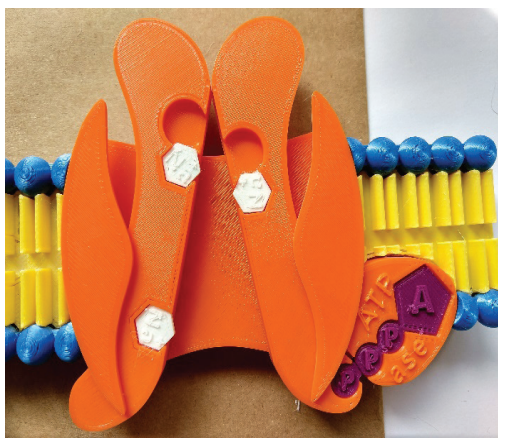

E)

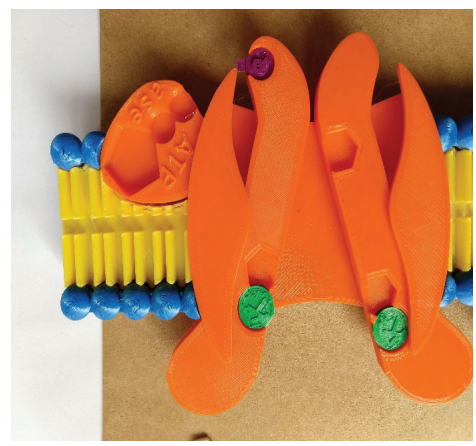


F)

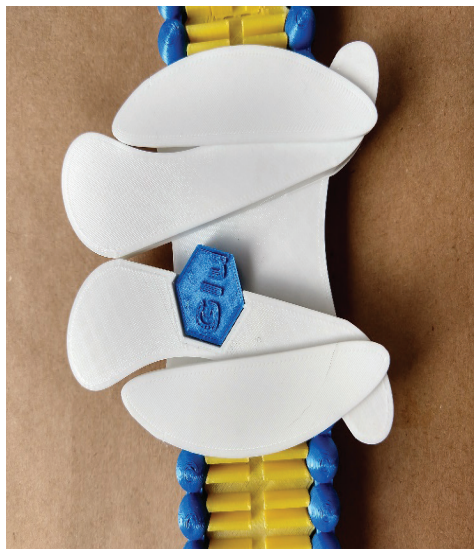

G)

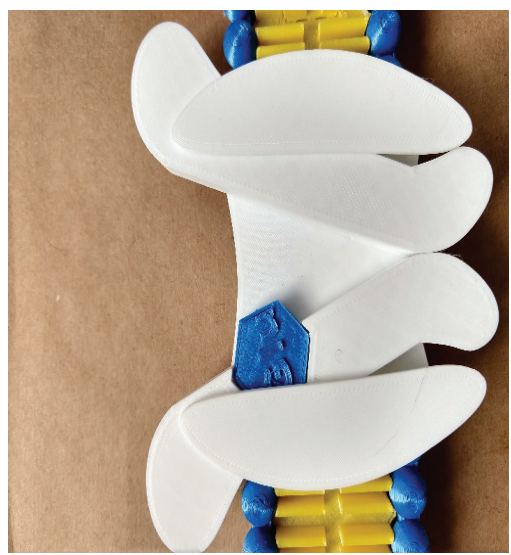

Figura 1 - Recurso educacional articulado para o ensino de transportes de membrana. A. Bicamada lipídica; B. Espaços para encaixe de proteínas; C. Proteínas encaixadas; D. Bomba de Sódio e Potássio no Estágio de captação de sódio; E. Bomba de sódio e potássio no estágio de captação de potássio; F. Proteínas Carreadora de Glicose aberta para o meio intracelular. G. Proteínas Carreadora de Glicose aberta para o meio extracelular.

\section{Ganho de vida com uso de microcontroladores}

O próximo passo na otimização dos modelos tridimensionalmente impressos para seu uso no ensino de processos e função, seria a adição de um recurso tecnológico capaz de conferir animação e responsividade aos modelos. Nesse contexto, entram os microcontroladores. Nos últimos cinco anos pudemos observar um aumento significativo no número de dispositivos nessa categoria, embarcando recursos como acesso à rede sem fio, bluetooth, baixo consumo de energia e baixo custo. Atualmente, o mercado conta com inúmeras opções, variando em poder de processamento e preços. Partimos de dispositivos relativamente simples e de baixo custo, baseados em chips da linha ATmega e PIC da fabricante MICROCHIP $\AA$, como o Arduino (ALVES et al., 2013), passando por controladores 32-bits como o ESP8866 e o ESP32 da fabricante chinesa Espressif ${ }^{\circledR}$ (CACHICHI, 2018) até chegarmos a computadores miniaturizados como a linha Raspberry Piß (SILVA; SÁ; TEIXEIRA, 2015). 
Com a utilização de microcontroladores, torna-se possível criar situações condicionadas em que determinadas ações do estudante geram respostas específicas no recurso educacional. Uma vez que os microcontroladores apresentam diferentes formas para a entrada de dados, a interação do estudante com a peça pode se dar por diferentes vias. $\mathrm{Na}$ visão mais simples, a interface pode ser gerada a partir de botões mecânicos que geram sinais que alimentam o sistema. Abordagens mais atuais podem ser obtidas a partir de sensores - presença, luminosidade, sinais biológicos - ou até mesmo a partir de internet das coisas, onde a peça é integrada a uma rede e controlada via aplicativos de celular (OLIVEIRA, 2017).

Atualmente, estamos trabalhando em dois protótipos envolvendo REs 3D microcontrolados. No primeiro, temos um neurônio controlado por um aplicativo de celular. O RE será utilizado para o ensino dos mecanismos de processamento de informação pela célula nervosa, bem como, dos processos de somação temporal e espacial. Via aplicativo o estudante pode fornecer informações ao neurônio por meio de diferentes vias de entrada (sinapses) e visualizar o seu efeito na geração de potenciais de ação dependendo da sua distância do cone axonal e na temporalidade do estímulo (KANDEL et al., 2015). Uma demonstração do protótipo pode ser encontrada em: https://youtu.be/o1oX-eaGWa8. Acesso em 12/11/2018.

No segundo protótipo, estamos desenvolvendo um modelo de coração impresso em material flexível. Cada câmara cardíaca (átrios e ventrículos) será conectada a um servomotor. Por meio de um aplicativo de celular, o aluno poderá selecionar diferentes traçados do eletrocardiograma e transmiti-los ao microcontrolador presente na peça. Este, por sua vez, irá ativar os servomotores de forma a simular o movimento cardíaco associado ao traçado de ECG selecionado pelo estudante.

\section{Adição de camada de realidade aumentada}

$\mathrm{O}$ uso de microcontroladores, descrito na subseção anterior, requer conhecimentos de eletrônica e investimento em hardware. Embora seu custo seja relativamente baixo, pode ser visto como um impedimento 
para sua aplicação na rotina de desenvolvimento de REs para ensino. Com isso em mente, temos como passo seguinte no desenvolvimento dos nossos REs o uso de aplicativos baseados em realidade aumentada (AR). Essa abordagem tecnológica vem ganhando espaço e se tornando cada vez mais acessível.

Diversas plataformas de desenvolvimento para AR estão disponíveis, incluindo alternativas gratuitas e de baixo custo. Entre os recursos com opção gratuita destacamos a Vuforia + Unity (For Beginers) e ARCore lançado pela Google ${ }^{\circledR}$ em agosto deste ano.

$\mathrm{O}$ uso de aplicativos baseados em AR nos permite criar uma camada adicional de realidade sobre o RE 3D, favorecendo a interação com o estudante. Recursos como animação, interatividade e exibição de informações em uma sequência temporal específica e aprendizadodependente podem ser implementados, criando recursos que respondem de forma personalizada, considerando as características do estudante. Um RE desenvolvido para o ensino de um conteúdo morfológico poderá então ser utilizado no ensino de função e no desenvolvimento de raciocínio clínico e pensamento crítico (FROST; DELANEY; FITZGERALD, 2017; KELLY et al., 2018).

\section{Considerações finais}

$\mathrm{O}$ uso de REs 3D para o ensino de CBS vem ganhando espaço. Um grupo crescente de profissionais têm se dedicado a pesquisar seus desenvolvimento e aplicação no ensino, validando seu uso em múltiplas estâncias. Uma deficiência que ainda precisa ser vencida emerge do fato de que tal tecnologia tem sido vista como um recurso apenas para o ensino de forma. Usar RE 3D para ensino de morfologia é uma abordagem lógica, imediatamente visualizada e motivada pelas características inerentes ao modelo. No entanto, restringir seu uso apenas ao ensino da forma é subutilizar a tecnologia.

Acreditamos que, com investimento no aprendizado de novas tecnologias, os docentes que estiverem interessados em dar o passo seguinte no uso de REs 3D, construindo-os de forma a permitir o ensino 
de função e processos biológicos, podem encontrar um caminho válido no uso de tecnologias como microcontroladores, softwares de desenho para engenharia e arquitetura e aplicativos de realidade aumentada. REs 3D são recursos impressionantes, apresentando potencial para revolucionar o ensino de CBS. No entanto, os docentes se encontram ainda nas fases iniciais de compreensão do potencial dessa tecnologia, sendo necessário aprofundar a discussão e o compartilhamento de ideias para que seu potencial seja melhor aproveitado.

\section{Referências}

ALVES, R. M. et al. Uso do Hardware Livre Arduino em Ambientes de Ensinoaprendizagem. Jornada de Atualização em Informática na Educação, v. 1, n. 1, p. 162-187, fev. 2013.

AUGUSTO, I. et al. Virtual reconstruction and three-dimensional printing of blood cells as a tool in cell biology education. PLoS ONE, v. 11, n. 8, p. 1-13, 2016.

BACKHOUSE, S.; TAYLOR, D.; ARMITAGE, J. A. Is This Mine to Keep? Three-dimensional Printing Enables Active, Personalized Learning in Anatomy. Anatomical Sciences Education, p. 1-11, 8 nov. 2018.

BAGLEY, J. R.; GALPIN, A. J. Three-dimensional printing of human skeletal muscle cells: An interdisciplinary approach for studying biological systems. Biochemistry and molecular biology education : a bimonthly publication of the International Union of Biochemistry and Molecular Biology, v. 43, n. 6, p. 403-407, 2015.

BANNON, R. et al. 3D printing the pterygopalatine fossa: a negative space model of a complex structure. Surgical and Radiologic Anatomy, v. 40, p. 185-191, 2018.

BRASIL. RESOLUÇÃO CNE/CES 2, DE 18 DE FEVEREIRO DE 2003. 2003.

CACHICHI, R. C. Uma nova proposta para experimentos remotos de Físico-Química utilizando microcontroladores e plataforma Moodle ${ }^{\circledR}$. [s.l.] Unicamp, 2018.

CANOVA, F.; SILVA, P.; GRASSI-KASSISSE, D. Alternativas para a diminuição do uso de animais na educação. Revista Neurociências, v. 23, n. 2, p. 313-316, jun. 2015.

CANTÍN, M.; MUÑOZ, M.; OLATE, S. Generation of 3D Tooth Models Based on Three-dimensional Scanning to Study the Morphology of Permanent TeethInt. J. Morphol. [s.l: s.n.]. Disponível em: <https://scielo. conicyt.cl/pdf/ijmorphol/v33n2/art57.pdf>. Acesso em: 12 nov. 2018. 
CARNIATTO, C. H. DE O. Propostas pedagógicas substitutivas ao uso de animais no ensino superior: uma revisão. Pubvet, v. 11, n. 5, p. 443-451, mai. 2017.

CASTANHA, D.; CASTRO, M. B. A Necessidade de Refletir sobre as Estratégias Pedagógicas para Atender à Aprendizagem da Geração Y. Revista de Educação do Cogeime, v. 19, n. 36, p. 27-38, jun. 2010.

CHEN, S. et al. The role of three-dimensional printed models of skull in anatomy education: A randomized controlled trail. Scientific Reports, v. 7, n. 1, p. 1-11, 2017.

DAVENPORT, J. et al. A Self-Assisting Protein Folding Model for Teaching Structural Molecular Biology. Structure, v. 25, n. 4, p. 671-678, 2017.

FELIX, J. et al. Geração C: Conectados em novos modelos de aprendizagem. VIII Brazilian Symposium on Games and Digital Entertainment. Anais... 2009.

FROST, J.; DELANEY, L.; FITZGERALD, R. University of Canberra implementing augmented reality into nursing education. [s.l: s.n.].

GARAS, M. et al. 3D-Printed specimens as a valuable tool in anatomy education: A pilot study. Annals of Anatomy, v. 219, p. 57-64, 1 set. 2018.

GOLDMAN, A. D.; BEATTY, J. T.; LANDWEBER, L. F. The TIM Barrel Architecture Facilitated the Early Evolution of Protein-Mediated Metabolism. Journal of Molecular Evolution, v. 82, n. 1, p. 17-26, 2016.

KANDEL, E. et al. Princípios de Neurociênciasitle, 5. ed. Porto Alegre: Grupo A, 2015.

KELLY, D. et al. Augmented reality learning environment for physiotherapy education. Physical Therapy Reviews, v. 23, n. 1, p. 21-28, jan. 2018.

KONG, X. et al. Do 3D Printing Models Improve Anatomical Teaching About Hepatic Segments to Medical Students? A Randomized Controlled Study. World Journal of Surgery, v. 40, n. 8, p. 1969-1976, 12 ago. 2016.

LEDERLE, F. et al. Improved mechanical properties of 3D-printed parts by fused deposition modeling processed under the exclusion of oxygen. Progress in Additive Manufacturing, v. 1, n. 1-2, p. 3-7, jun. 2016.

LI, F. et al. Production of accurate skeletal models of domestic animals using three-dimensional scanning and printing technology. Anatomical Sciences Education, v. 11, n. 1, p. 73-80, 1 . jan. 2018.

LIM, K. H. A. et al. Use of 3D printed models in medical education: A randomized control trial comparing 3D prints versus cadaveric materials for learning external cardiac anatomy. Anatomical Sciences Education, v. 9, n. 3, p. 213-221, mai. 2016.

LIMA, F. T. DE; STURN, R. M.; RIBEIRO, A. R. B. USE OF ANIMALS IN TEACHING VETERINARY MEDICINE: SUBSTITUTIVE METHODS. Nucleus, v. 15, n. 2, p. 251-264, out. 2018. 
MANUFACTUR3D. A Comprehensive List of All 3D Printing Technologies MANUFACTUR3D, nov. 2018.

MCMENAMIN, P. G. et al. The production of anatomical teaching resources using three-dimensional (3D) printing technology. Anatomical Sciences Education, v. 7, n. 6, p. 479-486, 2014.

OLIVEIRA, S. DE. Internet das Coisas com ESP8266, Arduino e Raspberry Pi. [s.l: s.n.].

PAUKSTELIS, P. J. MolPrint3D: Enhanced 3D Printing of Ball-and-Stick Molecular Models. Journal of Chemical Education, v. 95, n. 1, p. 169-172, 2018.

SARAIVA, T. DE V. et al. Atendimento à Lei Arouca no Ensino de Farmacologia no Curso de Medicina, UFC, Sobral. Revista Brasileira de Educação Médica, v. 40, n. 1, p. 138-143, mar. 2016.

SILVA, A. M. E. DA; SÁ, E.; TEIXEIRA, J. Especificando Objeto de Aprendizagem para Raspberry Pi usando Design Instrucional. Anais temporários do LACLO 2015, v. 10 , n. 1 , p. 252 , out. 2015.

SMITH, C. F. et al. Take away body parts! An investigation into the use of 3D-printed anatomical models in undergraduate anatomy education. Anatomical Sciences Education, v. 11, n. 1, p. 44-53, 2018.

SMITH, D. P. Bringing experiential learning into the lecture theatre using 3D printed objects. F1000Research, v. 5, n. 0, p. 61, 2016.

SMITH, M. L.; JONES, J. F. X. Dual-extrusion 3D printing of anatomical models for education. Anatomical Sciences Education, v. 11, n. 1, p. 65-72, 2018.

STREICHER, J. L.; KINI, S. P.; STOFF, B. K. Innovative dermatopathology teaching in a resource-limited environment. Journal of the American Academy of Dermatology United States, mai. 2016.

WONG, K. V.; HERNANDEZ, A. A Review of Additive Manufacturing. ISRN Mechanical Engineering, v. 2012, p. 1-10, 2012.

Submetido em: 15-10-2018

Aceito em: 30-11-2018 